\title{
The Viscosity Coefficients of Biaxial-Nematic Liquid Crystals. Phenomenology and Affine Transformation Model
}

\author{
Dieter Baalss \\ Institut für Theoretische Physik der Technischen Universität Berlin, Berlin
}

Z. Naturforsch. 45a, 7-13 (1990); received October 28, 1989

\begin{abstract}
The viscosity tensor of biaxial-nematic liquid crystals contains 16 independent elements. A complete set of these viscosity coefficients is introduced and related to experimentally accessible quantities. Furthermore, the flow alignment and its stability against an arbitrary infinitesimal disturbance are discussed. By the affine transformation model, formerly established for the uniaxial symmetric case, one can express the viscous anisotropy of perfectly ordered biaxial ellipsoids in terms of the two viscosities of an isotropic reference system and the axes ratios of the nonspherical particles.
\end{abstract}

\section{Introduction}

Almost two decades ago, phase transitions between uniaxial- and biaxial-nematic order (space groups $\mathbf{R}^{3} \times \mathbf{D}_{\infty \mathrm{h}}$ and $\mathbf{R}^{3} \times \mathbf{D}_{2 \mathrm{~h}}$ ) have been conjectured both for thermotropic and for lyotropic liquid crystals $[1,2]$. A biaxial-nematic phase was clearly observed for the first time in the ternary system potassium laurate-1-decanol- $\mathrm{D}_{2} \mathrm{O}$ [3], and today we have increasing experimental evidence for biaxial states even in simple low-molecular thermotropic systems [4-6]. Whereas the phenomenological description of the viscous behaviour is well-known for uniaxial nematics [7-11], a complete generalization to the biaxial case is still missing. In the first part of the present paper, this phenomenological theory is established. In the second part, it is illustrated for a system of completely aligned ellipsoids, whose viscous anisotropy can be evaluated by the affine transformation model previously applied to ellipsoids of revolution $[11,12]$.

\section{Phenomenology}

\subsection{Description of the Alignment}

We introduce a molecule-fixed orthonormal base $\left\{\boldsymbol{u}_{1}, \boldsymbol{u}_{2}, \boldsymbol{u}_{3}\right\}$ and define two alignment tensors of rank two:

$$
\mathbf{a}_{i}=\sqrt{3 / 2}\left\langle{\overrightarrow{u_{i}}}_{i}\right\rangle, \quad i=1,2,
$$

where $\langle\ldots\rangle$ denotes an ensemble average, and the

Reprint requests to Prof. Dr. S. Hess, Fachbereich 4, Physik, Theoretische Physik, Technische Universität Berlin, Hardenbergstraße 36, D-1000 Berlin 12. symmetric traceless part of a second rank tensor $\mathbf{T}$ is

$$
\overline{\mathbf{T}}=\frac{1}{2}\left(\mathbf{T}+\mathbf{T}^{\mathbf{t}}\right)-\frac{1}{3}(\operatorname{Tr} \mathbf{T}) \boldsymbol{\delta} ;
$$

the superscript " $\mathrm{t}$ " refers to transposition, "Tr" means "trace", and $\boldsymbol{\delta}$ is the unit tensor. A third alignment tensor is not needed due to

$$
\sum_{i=1}^{3} \overrightarrow{\boldsymbol{u}_{i} \boldsymbol{u}_{i}}=\mathbf{0}
$$

Obviously, $\mathbf{a}_{1}$ and $\mathbf{a}_{2}$ have common (orthonormalized) eigenvectors $\boldsymbol{n}_{1}, \boldsymbol{n}_{2}, \boldsymbol{n}_{3}$, which correspond to the "principal axes of alignment". The point group $\mathbf{D}_{2 \mathrm{~h}}$ implies equivalence of $\boldsymbol{n}_{\boldsymbol{i}}$ and $-\boldsymbol{n}_{\boldsymbol{i}}$ for $i=1,2,3$. The alignment tensors can be split into uniaxial and planarbiaxial contributions:

with

$$
\mathbf{a}_{i}=\sqrt{3 / 2}\left(a_{i} \mathbf{u}+b_{i} \pi\right), \quad i=1,2,
$$

$$
\mathrm{u} \equiv \overline{\boldsymbol{n}_{1}} \boldsymbol{n}_{1}, \quad \pi \equiv \boldsymbol{n}_{2} \boldsymbol{n}_{2}-\boldsymbol{n}_{3} \boldsymbol{n}_{3} .
$$

If $\Phi, \Theta, \Psi$ stand for the Euler angles (defined according to Goldstein [13]) in the principal axes system, in which $\Phi$ and $\Theta$ are measured towards $\boldsymbol{n}_{2}$ and $\boldsymbol{n}_{1}$, respectively, the scalar order parameters and their relation to the parameters $S, T, U, V$ used by Straley [14] as well as $D=\frac{3}{2} U$ introduced by Alben, McColl, and Shih [15] read

$$
\begin{aligned}
& a_{1}=S=\left\langle 1-\frac{3}{2} \sin ^{2} \Theta\right\rangle, \\
& b_{1}=-\frac{1}{2} T=\left\langle\left(\sin ^{2} \Phi-\frac{1}{2}\right) \sin ^{2} \Theta\right\rangle, \\
& a_{2}=-\frac{1}{2}(S+D)=\frac{1}{2}\left\langle 3 \sin ^{2} \Theta \sin ^{2} \Psi-1\right\rangle, \\
& b_{2}=\frac{1}{4} T+\frac{1}{2} V=\left\langle\left(\sin ^{2} \Phi-\frac{1}{2}\right)\left(\cos ^{2} \Theta \sin ^{2} \Psi-\cos ^{2} \Psi\right)\right. \\
&\left.\qquad-\frac{1}{2} \sin 2 \Phi \cos \Theta \sin 2 \Psi\right\rangle .
\end{aligned}
$$

0932-0784 / $90 / 0100-0007 \$ 01.30 / 0$. - Please order a reprint rather than making your own copy. 
Notice that these four quantities are not completely independent because the triplets $(S, T, U)$ lie inside a pyramid with the apex $(1,0,0)$ and the base corners $\left(-\frac{1}{2}, 1,1\right),\left(-\frac{1}{2},-1,1\right),\left(-\frac{1}{2},-1,-1\right),\left(-\frac{1}{2}, 1,-1\right)$. Only for $a_{1}=1$, which implies $b_{1}=0$ and $a_{2}=-\frac{1}{2}$, $b_{2}$ is an independent order parameter. For $a_{1}<1$ the last term in $b_{2}$ vanishes, and the equation

$$
b_{2}=b_{1}\left[2\left(a_{1}+2\right)\left(a_{2}+2\right)-9\right] /\left[2\left(a_{1}-1\right)^{2}\right]
$$

is valid in this case. The corresponding relation between the parameters defined in $[14,15]$ is

$$
V=T D(S+2) /\left[2(S-1)^{2}\right] \text {. }
$$

If the particles are uniaxial, $a_{1}$ and $b_{1}$ suffice for a complete description of the alignment.

\subsection{Calculation of the Number of Linear Viscosity Coefficients}

The viscosity tensor $\boldsymbol{\eta}$ is of rank four and connects the friction pressure tensor $\mathbf{p}$ with the velocity gradient $\nabla \boldsymbol{v}$. For reasons of objectivity, the latter has to be modified by a term taking into account the angular velocity $\boldsymbol{\Omega}$ of the principal axes system:

$$
\mathbf{p}=-2 \boldsymbol{\eta}:(\nabla v-\varepsilon \cdot \Omega)
$$

$\varepsilon$ is the totally antisymmetric isotropic tensor of rank three. In the eigenbase of the alignment tensors, the viscosity tensor has the representation

$$
\boldsymbol{\eta}=\sum_{i, j, k, l=1}^{3} \eta_{i j k l} \boldsymbol{n}_{i} \boldsymbol{n}_{j} \boldsymbol{n}_{k} \boldsymbol{n}_{l} .
$$

An elementary combinatorial consideration shows that 21 of the 81 elements of $\boldsymbol{\eta}$ are independent in the biaxial-nematic case. This number reduces to 9 for uniaxial-nematic and to 2 for spherical symmetry.

\subsection{Irreducible Representation of the Friction Pressure Tensor, Onsager Relations}

The number of 21 coefficients must be found again if we decompose the friction pressure tensor and the modified velocity gradient into their symmetric traceless, antisymmetric, and isotropic parts. We use the following abbreviations:

$$
\begin{aligned}
& \boldsymbol{\Gamma}:=\overline{\nabla \boldsymbol{v}}, \\
& \tilde{\boldsymbol{\Omega}}:=\boldsymbol{\varepsilon} \cdot(\boldsymbol{\Omega}-\boldsymbol{\omega}), \\
& \Delta:=\nabla \cdot \boldsymbol{v} ;
\end{aligned}
$$

$\omega \equiv \frac{1}{2} \nabla \times v$ is the vorticity of the flow field. The following ansatz contains all independent contributions:

$$
\begin{aligned}
& \mathbf{p}=-2\left(\eta \boldsymbol{\Gamma}+\eta_{u 2} \overline{\mathbf{u} \cdot \boldsymbol{\Gamma}}+\eta_{\pi 2} \overrightarrow{\pi \cdot \boldsymbol{\Gamma}}+\eta_{u 1} \overrightarrow{\mathbf{u} \cdot \tilde{\boldsymbol{\Omega}}}\right. \\
& +\eta_{\pi 1} \overline{\pi \cdot \tilde{\mathbf{\Omega}}}+\eta_{u 1 \pi} \overline{\mathbf{u} \cdot \tilde{\mathbf{\Omega}} \cdot \boldsymbol{\pi}}+\eta_{u}^{u} \mathbf{u} \mathbf{u}: \mathbf{\Gamma} \\
& \left.+\eta_{u}^{\pi} \mathbf{u} \pi: \boldsymbol{\Gamma}+\eta_{\pi}^{u} \pi \mathbf{u}: \mathbf{\Gamma}+\eta_{\pi}^{\pi} \pi \pi: \mathbf{\Gamma}\right) \\
& -\eta_{u}^{0} \mathbf{u} \Delta-\eta_{\pi}^{0} \boldsymbol{\pi} \Delta, \\
& \mathbf{p}^{\mathrm{a}}=\gamma \tilde{\boldsymbol{\Omega}}+\gamma_{u 1}(\mathbf{u} \cdot \tilde{\boldsymbol{\Omega}})^{\mathrm{a}}+\gamma_{\pi 1}(\boldsymbol{\pi} \cdot \tilde{\boldsymbol{\Omega}})^{\mathrm{a}}+\gamma_{u 2}(\mathbf{u} \cdot \boldsymbol{\Gamma})^{\mathrm{a}} \\
& +\gamma_{\pi 2}(\pi \cdot \Gamma)^{\mathrm{a}}+\gamma_{u 2 \pi}(\mathbf{u} \cdot \boldsymbol{\Gamma} \cdot \pi)^{\mathrm{a}}, \\
& p=-\varkappa \Delta-\chi^{u} \mathbf{u}: \boldsymbol{\Gamma}-\chi^{\pi} \pi: \boldsymbol{\Gamma},
\end{aligned}
$$

where the antisymmetric part of a second rank tensor $\mathbf{T}$ is

$$
\mathbf{T}^{\mathrm{a}}=\frac{1}{2}\left(\mathbf{T}-\mathbf{T}^{\mathrm{t}}\right)
$$

and $p \equiv \frac{1}{3} \operatorname{Tr} \mathbf{p}$; the notation used in (10) should speak for itself. Of course, all coefficients are functions of the order parameters (5). Taking into account the Onsager relations

$$
\begin{aligned}
& 2 \eta_{u 1}=\gamma_{u 2}, \quad 2 \eta_{\pi 1}=\gamma_{\pi 2}, \quad 2 \eta_{u 1 \pi}=\gamma_{u 2 \pi}, \\
& \eta_{u}^{0}=x^{u}, \quad \eta_{\pi}^{0}=x^{\pi},
\end{aligned}
$$

we establish a total of $21-5=16$ independent viscosity coefficients. The corresponding number for uniaxial nematics is $9-2=7$ [11].

\subsection{Definition of Experimentally Measurable Coefficients}

In order to obtain quantities which are, at least in principle, more or less directly accessible to the experiment, we shall now form linear combinations of the above viscosity coefficients. We restrict our attention to the shear, rotational, and normal pressure difference terms, disregarding the bulk and shear dilatancy viscosities.

\subsubsection{Shear Viscosities}

We presuppose a plane Couette flow with the constant shear rate $\Gamma$ :

$$
\nabla v=\Gamma \boldsymbol{e}^{y} e^{x} .
$$

$\boldsymbol{e}^{x, y, z}$ are unit vectors parallel to the $x$-, $y$-, and $z$-coordinate axes. It is understood that the mean angular velocity $\boldsymbol{\Omega}$ of the molecules vanishes. The shear viscosity $\eta_{\text {sh }}$ for an arbitrary orientation of the alignment 
tensors (1) is defined by

$$
p_{y x}=-\eta_{\mathrm{sh}} \Gamma \text {. }
$$

There are 9 independent values of $\eta_{\mathrm{sh}}$. We obtain a complete set of shear viscosities if we choose the 6 orientations where the principal axes of alignment coincide with the axes of the Couette geometry as well as 3 of the orientations where one principal axis lies in the neutral direction, i.e. in the $z$-axis, and the two others form an angle of $45^{\circ}$ with the flow and the velocity gradient direction, respectively. Our system of the pertaining viscosity coefficients, which is partly inspired by the Miesowicz notation for the uniaxial case [16], is listed in Table 1. As a generalization of the Helfrich coefficient $\eta_{12}$ [17] we furthermore define

$$
\begin{aligned}
& \eta_{12}:=4 \eta_{43}-2\left(\eta_{1}+\eta_{-2}\right), \\
& \eta_{23}:=4 \eta_{41}-2\left(\eta_{3}+\eta_{-3}\right), \\
& \eta_{31}:=4 \eta_{42}-2\left(\eta_{2}+\eta_{-1}\right) .
\end{aligned}
$$

Counting $\Phi$ from the velocity gradient and $\Theta$ from the flow direction, we ascertain the following expression for the shear viscosity as a function of the Euler angles $\Phi, \Theta, \Psi$ which describe the orientation of the principal axes of alignment with respect to the Couette geometry:

$$
\begin{gathered}
\eta_{\mathrm{sh}}=\frac{1}{2} \eta_{1} \cos \Theta[\cos \Theta(1+\cos 2 \Phi \cos 2 \Psi) \\
\left.-\frac{1}{2}\left(1+\cos ^{2} \Theta\right) \sin 2 \Phi \sin 2 \Psi\right] \\
+\frac{1}{2} \eta_{-1} \cos \Theta[\cos \Theta(1-\cos 2 \Phi \cos 2 \Psi) \\
\left.+\frac{1}{2}\left(1+\cos ^{2} \Theta\right) \sin 2 \Phi \sin 2 \Psi\right] \\
+\eta_{2} \sin ^{2} \Theta\left(\sin ^{2} \Phi \cos ^{2} \Psi+\frac{1}{4} \cos \Theta \sin 2 \Phi \sin 2 \Psi\right) \\
+\eta_{-2} \sin ^{2} \Theta\left(\sin ^{2} \Phi \sin ^{2} \Psi-\frac{1}{4} \cos \Theta \sin 2 \Phi \sin 2 \Psi\right) \\
+\eta_{3} \sin ^{2} \Theta\left(\cos ^{2} \Phi \sin ^{2} \Psi+\frac{1}{4} \cos \Theta \sin 2 \Phi \sin 2 \Psi\right) \\
+\eta_{-3} \sin ^{2} \Theta\left(\cos ^{2} \Phi \cos ^{2} \Psi-\frac{1}{4} \cos \Theta \sin 2 \Phi \sin 2 \Psi\right) \\
+\eta_{12} \sin ^{2} \Theta \cos \Theta\left(\cos \Theta \sin ^{2} \Phi \sin ^{2} \Psi\right.
\end{gathered}
$$$$
\left.-\frac{1}{4} \sin 2 \Phi \sin 2 \Psi\right)
$$$$
+\frac{1}{4} \eta_{23} \sin ^{2} \Theta \sin 2 \Psi[\cos \Theta \sin 2 \Phi \cos 2 \Psi
$$$$
\left.-\left(\cos ^{2} \Theta \sin ^{2} \Phi-\cos ^{2} \Phi\right) \sin 2 \Psi\right]
$$$$
+\eta_{31} \sin ^{2} \Theta \cos \Theta\left(\cos \Theta \sin ^{2} \Phi \cos ^{2} \Psi\right.
$$

$$
\left.+\frac{1}{4} \sin 2 \Phi \sin 2 \Psi\right) \text {. }
$$

If we make use of the Onsager relations (12), the shear viscosities are connected with the coefficients of the irreducible representation by

$$
\begin{aligned}
\eta_{1}= & \eta+\frac{1}{6} \eta_{u 2}+\frac{1}{2} \eta_{\pi 2}+\eta_{u 1}-\eta_{\pi 1}+\frac{2}{3} \eta_{u 1 \pi}+\frac{1}{2} \gamma \\
& +\frac{1}{12} \gamma_{u 1}+\frac{1}{4} \gamma_{\pi 1}, \\
\eta_{-1}= & \eta+\frac{1}{6} \eta_{u 2}-\frac{1}{2} \eta_{\pi 2}+\eta_{u 1}+\eta_{\pi 1}-\frac{2}{3} \eta_{u 1 \pi}+\frac{1}{2} \gamma \\
& +\frac{1}{12} \gamma_{u 1}-\frac{1}{4} \gamma_{\pi 1}, \\
\eta_{2}= & \eta+\frac{1}{6} \eta_{u 2}-\frac{1}{2} \eta_{\pi 2}-\eta_{u 1}-\eta_{\pi 1}+\frac{2}{3} \eta_{u 1 \pi}+\frac{1}{2} \gamma \\
& +\frac{1}{12} \gamma_{u 1}-\frac{1}{4} \gamma_{\pi 1}, \\
\eta_{-2}= & \eta+\frac{1}{6} \eta_{u 2}+\frac{1}{2} \eta_{\pi 2}-\eta_{u 1}+\eta_{\pi 1}-\frac{2}{3} \eta_{u 1 \pi}+\frac{1}{2} \gamma \\
& +\frac{1}{12} \gamma_{u 1}+\frac{1}{4} \gamma_{\pi 1}, \\
\eta_{3}= & \eta-\frac{1}{3} \eta_{u 2}+2 \eta_{\pi 1}+\frac{2}{3} \eta_{u 1 \pi}+\frac{1}{2} \gamma-\frac{1}{6} \gamma_{u 1}, \\
\eta_{-3}= & \eta-\frac{1}{3} \eta_{u 2}-2 \eta_{\pi 1}-\frac{2}{3} \eta_{u 1 \pi}+\frac{1}{2} \gamma-\frac{1}{6} \gamma_{u 1}, \\
\eta_{12}= & 2\left(\eta_{u}^{u}-\eta_{u}^{\pi}-\eta_{\pi}^{u}+\eta_{\pi}^{\pi}\right), \\
\eta_{23}= & 8 \eta_{\pi}^{\pi}, \\
\eta_{31}= & 2\left(\eta_{u}^{u}+\eta_{u}^{\pi}+\eta_{\pi}^{u}+\eta_{\pi}^{\pi}\right) .
\end{aligned}
$$

\subsubsection{Rotational Viscosities, Analogues of the Parodi Equation}

The density of the viscous torque, which corresponds to the antisymmetric part of the friction pressure tensor (10), is

$$
\begin{aligned}
\vartheta= & -\left(\gamma_{11} n_{1} n_{1}+\gamma_{12} n_{2} n_{2}+\gamma_{13} n_{3} n_{3}\right) \cdot(\Omega-\omega) \\
& -\left(\gamma_{21} n_{1} n_{2} n_{3}+\gamma_{22} n_{2} n_{3} n_{1}+\gamma_{23} n_{3} n_{1} n_{2}\right): \Gamma
\end{aligned}
$$

with the coefficients

$$
\begin{aligned}
& \gamma_{11}=2 \gamma-\frac{2}{3} \gamma_{u 1}, \\
& \gamma_{12}=2 \gamma+\frac{1}{3} \gamma_{u 1}-\gamma_{\pi 1}, \\
& \gamma_{13}=2 \gamma+\frac{1}{3} \gamma_{u 1}+\gamma_{\pi 1}, \\
& \gamma_{21}=2 \gamma_{\pi 2}+\frac{2}{3} \gamma_{u 2 \pi}, \\
& \gamma_{22}=-\gamma_{u 2}-\gamma_{\pi 2}+\frac{2}{3} \gamma_{u 2 \pi}, \\
& \gamma_{23}=\gamma_{u 2}-\gamma_{\pi 2}+\frac{2}{3} \gamma_{u 2 \pi} .
\end{aligned}
$$

The quantities (19) are the biaxial generalizations of the Leslie coefficients $\gamma_{1}$ and $\gamma_{2}: \gamma_{1 i}, i=1,2,3$, is the rotational viscosity for a rotation of the molecules around the principal axis pertaining to $\boldsymbol{n}_{i}$. The $\gamma_{2 i}$, $i=1,2,3$, allow to formulate the first three of the Onsager relations (12) on the analogy of the Parodi equation [18]:

$\gamma_{21}=\eta_{3}-\eta_{-3}, \quad \gamma_{22}=\eta_{2}-\eta_{-1}, \quad \gamma_{23}=\eta_{1}-\eta_{-2}$. 
Now we can invert (17) and (19):

$$
\begin{aligned}
\eta= & \frac{1}{6}\left[\eta_{1}+\eta_{-1}+\eta_{2}+\eta_{-2}+\eta_{3}+\eta_{-3}\right. \\
& \left.-\frac{1}{2}\left(\gamma_{11}+\gamma_{12}+\gamma_{13}\right)\right], \\
\eta_{u 2}= & \frac{1}{2}\left(\eta_{1}+\eta_{-1}+\eta_{2}+\eta_{-2}\right)-\eta_{3}-\eta_{-3}+\frac{1}{2} \gamma_{11} \\
& -\frac{1}{4}\left(\gamma_{12}+\gamma_{13}\right), \\
\eta_{\pi 2}= & \frac{1}{2}\left(\eta_{1}-\eta_{-1}-\eta_{2}+\eta_{-2}\right)+\frac{1}{4}\left(\gamma_{12}-\gamma_{13}\right), \\
\eta_{u 1}= & \frac{1}{4}\left(\eta_{1}+\eta_{-1}-\eta_{2}-\eta_{-2}\right), \\
\eta_{\pi 1}= & \frac{1}{6}\left[\eta_{3}-\eta_{-3}-\frac{1}{2}\left(\eta_{1}-\eta_{-1}+\eta_{2}-\eta_{-2}\right)\right], \\
\eta_{u 1 \pi}= & \frac{1}{4}\left(\eta_{1}-\eta_{-1}+\eta_{2}-\eta_{-2}+\eta_{3}-\eta_{-3}\right), \\
\eta_{u}^{u}= & \frac{1}{4}\left(\eta_{12}+\eta_{31}-\frac{1}{2} \eta_{23}\right), \\
\eta_{u}^{\pi}+ & \eta_{\pi}^{u}=\frac{1}{4}\left(\eta_{31}-\eta_{12}\right), \\
\eta_{\pi}^{\pi}= & \frac{1}{8} \eta_{23}, \\
\gamma^{=}= & \frac{1}{6}\left(\gamma_{11}+\gamma_{12}+\gamma_{13}\right), \\
\gamma_{u 1}= & \frac{1}{2}\left(\gamma_{12}+\gamma_{13}\right)-\gamma_{11}, \\
\gamma_{\pi 1}= & \frac{1}{2}\left(\gamma_{13}-\gamma_{12}\right), \\
\gamma_{u 2}= & \frac{1}{2}\left(\gamma_{23}-\gamma_{22}\right), \\
\gamma_{\pi 2}= & \frac{1}{3}\left[\gamma_{21}-\frac{1}{2}\left(\gamma_{22}+\gamma_{23}\right)\right], \\
\gamma_{u 2 \pi}= & \frac{1}{2}\left(\gamma_{21}+\gamma_{22}+\gamma_{23}\right) .
\end{aligned}
$$

We see that a prescription for the separate determination of $\eta_{u}^{\pi}$ and $\eta_{\pi}^{u}$ is still missing. This leads us to a quantity which has no uniaxial analogue. In the next section, we shall establish a suitable definition.

\subsubsection{Normal Pressure Difference Viscosity}

It is easily shown that normal pressure differences and transverse forces should occur even in uniaxial nematics, and the transverse pressure $p_{z y}$ was also verified experimentally [19]. In the axial symmetric case, these effects do not entail viscosity coefficients being independent of the shear and rotational viscosities, whereas they involve one additional quantity for the lower symmetry of biaxial nematics. In order to prove this statement, we set

$$
\eta_{0 i}:=\frac{1}{2}\left[\frac{1}{2}\left(p_{x x}+p_{y y}\right)-p_{z z}\right] / \Gamma, \quad i=1,2,3,
$$

for the same orientations in the plane Couette flow (13) which were used to define $\eta_{4 i}$ (cf. Table 1), and we call

$$
\eta_{0}:=\frac{1}{3}\left(\eta_{01}+\eta_{02}+\eta_{03}\right)
$$

Table 1. Definition of shear viscosity coefficients for 9 orientations of the eigenvectors $\boldsymbol{n}_{1}, \boldsymbol{n}_{2}, \boldsymbol{n}_{3}$ of the alignment tensors in a plane Couette flow with the velocity in the $x$-direction and its gradient in the $y$-direction.

\begin{tabular}{cccl}
\hline$n_{1}$ & $n_{2}$ & $n_{3}$ & $\begin{array}{l}\text { Shear } \\
\text { viscosity }\end{array}$ \\
\hline $\boldsymbol{e}^{x}$ & $\boldsymbol{e}^{y}$ & $\boldsymbol{e}^{z}$ & $\eta_{1}$ \\
$\boldsymbol{e}^{x}$ & $\boldsymbol{e}^{z}$ & $-\boldsymbol{e}^{y}$ & $\eta_{-1}$ \\
$\boldsymbol{e}^{y}$ & $\boldsymbol{e}^{z}$ & $\boldsymbol{e}^{x}$ & $\eta_{2}$ \\
$\boldsymbol{e}^{y}$ & $\boldsymbol{e}^{x}$ & $-\boldsymbol{e}^{z}$ & $\eta_{-2}$ \\
$\boldsymbol{e}^{z}$ & $\boldsymbol{e}^{x}$ & $\boldsymbol{e}^{y}$ & $\eta_{3}$ \\
$\boldsymbol{e}^{z}$ & $\boldsymbol{e}^{y}$ & $-\boldsymbol{e}^{x}$ & $\eta_{-3}$ \\
\hline $\boldsymbol{e}^{z}$ & $\left(\boldsymbol{e}^{x}+\boldsymbol{e}^{y}\right) / \sqrt{2}$ & $\left(\boldsymbol{e}^{y}-\boldsymbol{e}^{x}\right) / \sqrt{2}$ & $\eta_{41}$ \\
$\left(\boldsymbol{e}^{y}-\boldsymbol{e}^{x}\right) / \sqrt{2}$ & $\boldsymbol{e}^{z}$ & $\left(\boldsymbol{e}^{x}+\boldsymbol{e}^{y}\right) / \sqrt{2}$ & $\eta_{42}$ \\
$\left(\boldsymbol{e}^{x}+\boldsymbol{e}^{y}\right) / \sqrt{2}$ & $\left(\boldsymbol{e}^{y}-\boldsymbol{e}^{x}\right) / \sqrt{2}$ & $\boldsymbol{e}^{z}$ & $\eta_{43}$ \\
\hline
\end{tabular}

the "normal pressure difference viscosity". The equations

$$
\begin{aligned}
\eta_{01}=\eta_{0}+\frac{1}{4}[ & \eta_{2}+\eta_{-1}-\eta_{1}-\eta_{-2} \\
+ & \left.\frac{1}{2}\left(\eta_{31}-\eta_{12}-\gamma_{12}+\gamma_{13}\right)\right], \\
\eta_{02}=\eta_{0}+\frac{1}{4}[ & \eta_{1}+\eta_{-2}-\eta_{3}-\eta_{-3} \\
& \left.+\frac{1}{2}\left(\eta_{12}-\eta_{23}-\gamma_{13}+\gamma_{11}\right)\right], \\
\eta_{03}=\eta_{0}+\frac{1}{4}[ & \eta_{3}+\eta_{-3}-\eta_{2}-\eta_{-1} \\
& \left.+\frac{1}{2}\left(\eta_{23}-\eta_{31}-\gamma_{11}+\gamma_{12}\right)\right],
\end{aligned}
$$

and

$$
\eta_{0}=\frac{1}{2}\left(\eta_{u}^{\pi}-\eta_{\pi}^{u}\right)
$$

are readily deduced. By comparison of (25) with (21) we see that $\eta_{0}$ is indeed an independent viscosity coefficient, and we can write down separate expressions for $\eta_{u}^{\pi}$ and $\eta_{\pi}^{u}$ :

$$
\begin{aligned}
& \eta_{u}^{\pi}=\frac{1}{8}\left(\eta_{31}-\eta_{12}\right)+\eta_{0}, \\
& \eta_{\pi}^{u}=\frac{1}{8}\left(\eta_{31}-\eta_{12}\right)-\eta_{0} .
\end{aligned}
$$

\subsection{Flow Alignment}

We are looking for orientations of the alignment tensors (1) with respect to the plane Couette flow (13) which lead to a vanishing torque density (18). For symmetry reasons, one principal axis of alignment must coincide with the neutral direction in each of these stationary states. First we regard the case that the concerning axis is fixed, and then we analyse the stability against release from this constraint. 
1.5.1. Flow A lignment Angle for Fixed Principal Axis in Neutral Direction

If $\boldsymbol{n}_{i}$ is fixed and $\vartheta_{0 i}$ denotes the alignment angle of $\boldsymbol{n}_{j}$, where $(i, j, k)$ is a cyclic permutation of $(1,2,3)$, the condition for $\boldsymbol{\vartheta}=\mathbf{0}$ reads

$$
\cos 2 \vartheta_{0 i}=-\gamma_{1 i} / \gamma_{2 i} \text {. }
$$

In the case of $\left|\gamma_{2 i}\right|>\gamma_{1 i}$, this corresponds to two real angles. The stable solution is

$$
\vartheta_{0 i}=\frac{1}{2}\left\{\pi+\left[\operatorname{sgn}\left(\gamma_{2 i} \Gamma\right)\right] \arccos \left(\gamma_{1 i} / \gamma_{2 i}\right)\right\} .
$$

A measurement of the angle (28) renders it possible to examine the relevant one of the Parodi equations (20).

\subsubsection{Stability against Free Rotation}

We investigate an infinitesimal disturbance of the flow alignment with $\boldsymbol{n}_{\boldsymbol{k}}$ lying in the neutral direction. Due to the nematic symmetry we can assume that the principal axes of inertia and alignment coincide. The analysis of the stability in Lyapunov's sense leads to the quadratic equation

$$
\lambda^{2}+c_{1} \lambda+c_{0}=0
$$

with

$c_{0}=\left(\Gamma^{2} / 4\right)\left[\gamma_{2 i} \gamma_{2 j} h /\left(\Theta_{i} \Theta_{j}\right)\right] \sin \vartheta_{0 k}$,

$c_{1}=(\Gamma / 2)\left[\left(\gamma_{2 i} / \Theta_{i}\right)+\left(\gamma_{2 \mathrm{j}} / \Theta_{j}\right) \sin \vartheta_{0 k}\right] \sin 2 \vartheta_{0 k}$,

where $i, j, k$ are cyclic indices, $\Theta_{i}$ is the density of the momentum of inertia around $\boldsymbol{n}_{i}$, and

$$
\begin{aligned}
h \equiv 1 & +\gamma_{11} \gamma_{12} /\left(\gamma_{21} \gamma_{22}\right)+\gamma_{12} \gamma_{13} /\left(\gamma_{22} \gamma_{23}\right) \\
& +\gamma_{13} \gamma_{11} /\left(\gamma_{23} \gamma_{21}\right)
\end{aligned}
$$

is a harmonic function of the arguments $\gamma_{11} / \gamma_{21}$, $\gamma_{12} / \gamma_{22}, \gamma_{13} / \gamma_{23}$. A sufficient condition of both roots having a negative real part and consequently of stability is

$$
\operatorname{sgn} \gamma_{2 i}=\operatorname{sgn} \gamma_{2 j}=-\operatorname{sgn} \gamma_{2 k} \wedge h>0 .
$$

If one part of the condition is fulfilled, the other is necessary.

The existence of three real flow alignment angles (28) for fixed principal axis implies $\gamma_{1 i} /\left|\gamma_{2 i}\right|<1$ $(i=1,2,3)$. In the pertaining cube, the harmonic function (31) has positive values, and then because of the first part of the condition (32) at most one solution can remain stable at release of the principal axis.

However, if there are only two real flow alignment angles for fixed principal axis, $h<0$ can be valid, and in this case it is possible that both solutions are stable against an arbitrary infinitesimal disturbance.

\section{Affine Transformation Model}

\subsection{Fundamentals}

For the case of uniaxial-nematic (including nematic discotic) liquid crystals, the full particulars of our affine transformation model and its test via non-equilibrium molecular dynamics simulations have been reported in $[11,12]$. The generalization for biaxial ellipsoids is straightforward.

We investigate an interaction potential $\Phi$ whose equipotential surfaces are given by the relation

$$
\mathbf{A}: \boldsymbol{r} \boldsymbol{r} \equiv r_{\mathrm{A}}^{2}=\text { const }
$$

with the unimodular matrix

$$
\mathbf{A}=\sum_{i=1}^{3} A_{i} \boldsymbol{u}_{i} \boldsymbol{u}_{i}
$$

where the $\boldsymbol{u}_{i}$ form an orthonormal system and the eigenvalues $A_{i}$ are positive; $\boldsymbol{r}$ stands for the relative position vector of two particles. Equation (33) describes an ellipsoid with the semiaxes

$$
a=r_{\mathrm{A}} / \sqrt{A_{1}}, \quad b=r_{\mathrm{A}} / \sqrt{A_{2}}, \quad c=r_{\mathrm{A}} / \sqrt{A_{3}} ;
$$

the symmetry axes lie in the directions of the $\boldsymbol{u}_{i}$. Such a system serves as a model of a biaxial-nematic liquid crystal with the order parameters (cf. (5))

$$
a_{1}=1, \quad b_{1}=0, \quad a_{2}=-\frac{1}{2}, \quad b_{2}=\frac{1}{2}
$$

or, equivalently,

$$
S=1, T=0, \quad U=0, \quad V=1 .
$$

In the affine coordinates

$$
\boldsymbol{r}_{\mathrm{A}} \equiv \mathbf{A}^{\frac{1}{2}} \cdot \boldsymbol{r},
$$

$\Phi$ appears as a central potential:

$$
\Phi=\Phi\left(r_{\mathrm{A}}\right) .
$$

Assuming that the pair-correlation function does not depend on the $\boldsymbol{u}_{i}$ in the affine coordinates (37), the potential contribution to the friction pressure tensor is $\mathbf{p}^{\text {pot }}=-\eta_{\text {iso }}^{\text {pot }}\left[\mathbf{A}^{-1} \cdot \nabla \boldsymbol{v} \cdot \mathbf{A}+(\nabla \boldsymbol{v})^{\mathrm{t}}-\frac{2}{3} \nabla \cdot \boldsymbol{v} \boldsymbol{\delta}\right]-\varkappa_{\text {iso }} \nabla \cdot \boldsymbol{v} \boldsymbol{\delta}$,

where $\eta_{\text {iso }}^{\text {pot }}$ and $\varkappa_{\text {iso }}$ denote the (potential) shear and bulk viscosity of the isotropic reference system. 


\subsection{Results}

In the following, the superscript "pot" is omitted, and we use the abbreviations

$$
Q_{1}:=b / c, \quad Q_{2}:=c / a, \quad Q_{3}:=a / b
$$

as well as

$D_{i}:=\left(Q_{i}-Q_{i}^{-1}\right)^{2} \eta_{\text {iso }}, \quad d_{i}:=\left(Q_{i}^{2}-Q_{i}^{-2}\right) \eta_{\text {iso }}$,

From the equations of this section, one can derive the previously published results for ellipsoids of revolution with the axes ratio $Q \equiv a / b[11,12]$ by setting $b=c$ and consequently $Q_{1}=1, Q_{2}=Q^{-1}, Q_{3}=Q$.

The biaxial affine transformation yields for the coefficients of the ansatz (10)

$$
\begin{aligned}
& \eta=\eta_{\text {iso }}+\frac{1}{12}\left(D_{1}+D_{2}+D_{3}\right), \\
& \eta_{u 2}=\frac{1}{2}\left[\frac{1}{2}\left(D_{2}+D_{3}\right)-D_{1}\right] \text {, } \\
& \eta_{\pi 2}=\frac{1}{4}\left(D_{3}-D_{2}\right) \text {, } \\
& \eta_{u 1}=\frac{1}{4}\left(d_{2}-d_{3}\right) \text {, } \\
& \eta_{\pi 1}=\frac{1}{6}\left[\frac{1}{2}\left(d_{2}+d_{3}\right)-d_{1}\right], \\
& \eta_{u 1 \pi}=-\frac{1}{4}\left(d_{1}+d_{2}+d_{3}\right) \text {, } \\
& \eta_{u}^{u}=\frac{1}{4}\left(\frac{1}{2} D_{1}-D_{2}-D_{3}\right), \\
& \eta_{u}^{\pi}=\eta_{\pi}^{u}=\frac{1}{8}\left(D_{3}-D_{2}\right), \\
& \eta_{\pi}^{\pi}=-\frac{1}{8} D_{1} \text {, } \\
& \eta_{u}^{0}=\eta_{\pi}^{0}=0 \text {, } \\
& \gamma=\frac{1}{6}\left(D_{1}+D_{2}+D_{3}\right), \\
& \gamma_{u 1}=\frac{1}{2}\left(D_{2}+D_{3}\right)-D_{1} \text {, } \\
& \gamma_{\pi 1}=\frac{1}{2}\left(D_{3}-D_{2}\right) \text {, } \\
& \gamma_{u 2}=\frac{1}{2}\left(d_{2}-d_{3}\right) \text {, } \\
& \gamma_{\pi 2}=\frac{1}{3}\left[\frac{1}{2}\left(d_{2}+d_{3}\right)-d_{1}\right], \\
& \gamma_{u 2 \pi}=-\frac{1}{2}\left(d_{1}+d_{2}+d_{3}\right) \text {, } \\
& \varkappa=\chi_{\text {iso }}, \quad \chi^{u}=\chi^{\pi}=0 \text {. }
\end{aligned}
$$

Notice that all the five Onsager relations (12) are fulfilled.
The "experimental" coefficients of Section 1.4 are

$$
\begin{aligned}
& \eta_{1}=Q_{3}^{-2} \eta_{\text {iso }}, \\
& \eta_{-1}=Q_{2}^{2} \eta_{\text {iso }}=Q_{1}^{-2} \eta_{1}, \\
& \eta_{2}=Q_{2}^{-2} \eta_{\text {iso }}, \\
& \eta_{-2}=Q_{3}^{2} \eta_{\text {iso }}=Q_{1}^{-2} \eta_{2}, \\
& \eta_{3}=Q_{1}^{-2} \eta_{\text {iso }}, \\
& \eta_{-3}=Q_{1}^{2} \eta_{\text {iso }}=Q_{1}^{4} \eta_{3}, \\
& \eta_{i j}=-\left(Q_{k}-Q_{k}^{-1}\right)^{2} \eta_{\text {iso }}, \\
& \gamma_{1 i}=\left(Q_{i}-Q_{i}^{-1}\right)^{2} \eta_{\text {iso }}=-\eta_{j k}, \\
& \gamma_{2 i}=\left(Q_{i}^{-2}-Q_{i}^{2}\right) \eta_{\text {iso }}, \\
& \eta_{0 i}=\eta_{0}=0
\end{aligned}
$$

with $i=1,2,3$ and cyclic indices $i, j, k$. The quotients $\eta_{i} / \eta_{\text {iso }}$ and $\eta_{-i} / \eta_{\text {iso }}$ equal in each case the squared ratio of the semiaxis in the gradient direction to the semiaxis in the flow direction. Not unexpectedly, the smallest (largest) values of the generalized Miesowicz viscosity are found for those orientations where the largest (smallest) semiaxis is parallel to the flow and the shortest (largest) semiaxis is parallel to the velocity gradient. Whereas the $\eta_{i}, \eta_{-i}$, and $\gamma_{1 i}$ are positive, which is also necessary for thermodynamic reasons, the $\gamma_{2 i}$ may have either sign; for $a>b>c$ one has $\gamma_{21}<0, \gamma_{22}>0, \gamma_{23}<0$. From (46) we see that the quantity which determines the flow alignment angle according to (27) is

$$
\gamma_{1 i} / \gamma_{2 i}=\left(1-Q_{i}^{2}\right) /\left(1+Q_{i}^{2}\right) .
$$

Therefore the inequations $\left|\gamma_{2 i}\right|>\gamma_{1 i}, i=1,2,3$, are valid for biaxial ellipsoids. Condition (32) in connection with (28) and the definitions (40) shows that the flow alignment is stable against free rotation just if the medium semiaxis coincides with the neutral direction and if the largest (smallest) semiaxis lies in the 1st and 3rd quadrant of the Couette plane for positive (negative) shear rate $\Gamma$.

\section{Concluding Remarks}

In this article, we have demonstrated under which experimental geometries the shear, rotational, and normal pressure difference viscosities of biaxial nematics could be measured, and we have shown how the 
analogues of the Parodi equation could be verified. Of course, such experiments require to orient the principal axes of the liquid crystal in the desired way, and this might be a serious difficulty for the practical measurement of the complete set of viscosities and of the three flow alignment angles. However, it is certainly possible to fix at least one axis and so to determine e.g. the arithmetic mean values of $\eta_{1}$ and $\eta_{-1}$ or of $\gamma_{12}$ and $\gamma_{13}$. As regards the affine transformation model, our

[1] M. J. Freiser, Mol. Cryst. Liq. Cryst. 14, 165 (1971).

[2] R. Alben, J. Chem. Phys. 59, 4299 (1973).

[3] L. J. Yu and A. Saupe, Phys. Rev. Lett. 45, 1000 (1980).

[4] J. Malthête, L. Liébert, A.-M. Levelut, and Y. Galerne, C. R. Acad. Sci. Paris 303, 1073 (1986).

[5] S. Chandrasekhar, B. K. Sadashiva, B. R. Ratna, and V. N. Raja, Pramana 30, L 491 (1988).

[6] K. Praefcke, B. Kohne, B. Gündoğan, D. Demus, and S. Diele, 18. Freiburger Arbeitstagung Flüssigkristalle (1989).

[7] M. J. Stephen and J. P. Straley, Rev. Mod. Phys. 46, 617 (1974).

[8] P. G. de Gennes, The Physics of Liquid Crystals, Clarendon Press, Oxford 1974.

[9] H. Kelker and R. Hatz, Handbook of Liquid Crystals, Verlag Chemie, Weinheim 1980. remarks on its touch with reality in [11] apply by analogy to the biaxial case.

\section{Acknowledgements}

This research has been conducted under the auspices of the Sonderforschungsbereich 335 "Anisotrope Fluide". Helpful discussions with Professor Dr. Siegfried Hess and Professor Dr. Wolfgang Muschik are gratefully acknowledged.

[10] S. Hess, J. Non-Equilib. Thermodyn. 11, 176 (1986).

[11] D. Baalss and S. Hess, Phys. Rev. Lett. 57, 86 (1986); Z. Naturforsch. 43 a, 662 (1988).

[12] H. Sollich, D. Baalss, and S. Hess, Mol. Cryst. Liq. Cryst. 168, 189 (1989).

[13] H. Goldstein, Klassische Mechanik, Akademische Verlagsgesellschaft, Wiesbaden 1976.

[14] J. P. Straley, Phys. Rev. A 10, 1881 (1974).

[15] R. Alben, J. R. McColl, and C. S. Shih, Solid State Commun. 11, 1081 (1972).

[16] M. Miesowicz, Bull. Int. Acad. Polon., Sci. Lett. Ser. A 1936, 228 (1936); Nature London 158, 27 (1946).

[17] W. Helfrich, J. Chem. Phys. 51, 4092 (1969).

[18] O. Parodi, J. Phys. (Paris) 31, 581 (1970).

[19] P. Pieranski and E. Guyon, Phys. Lett. 49 A, 237 (1974). 